\title{
Lamb congenital lymphatic malformation - a case report
}

\author{
Neria Santos ${ }^{1}$, Luis Miguel Ferrer ${ }^{2}$, Delia Lacasta², Juan José Ramos², Alicia Uixera², \\ María Climent $^{2}$, Luis Figueras ${ }^{2}$
}

\begin{abstract}
${ }^{1}$ Rural Federal University of Pernambuco, Veterinary Medicine Department, Pernambuco, Brazil
${ }^{2}$ University of Zaragoza, Veterinary Faculty, Animal Pathology Department, Zaragoza, Spain
\end{abstract}

Received December 13, 2013

Accepted May 28, 2014

\begin{abstract}
Lymphatic malformations have been rarely reported in literature either in humans or in animals. However, in recent years, concern about these malformations in humans has increased. A five-month-old Rasa Aragonesa male lamb was received at the Ovine Clinical Service of the Veterinary Faculty of Zaragoza, Spain, with a history of cervical protuberances coming from birth. The lamb showed three round swollen lumps $(5-12 \mathrm{~cm}$ in diameter) parallel to the trachea on the left side of the neck. Clinical examination, haematology, ultrasonography, fluid examination and histopathology were performed. No abnormalities were found in blood samples and ultrasound confirmed a multicystic lesion with internal separations. Histological evaluation of the tissue revealed dilated lymphatic vessels and channels in the dermis and hypodermis; some lymphatic vessels were filled with amorphous proteinaceous material and occasional lymphocytes and macrophages. These protuberances were diagnosed as congenital lymphatic malformations. Most of the gross and microscopical lesions were very similar to those described in humans. To the authors' knowledge, this is the first time that a congenital lymphatic malformation is described in sheep.
\end{abstract}

Sheep, cervical protuberances, congenital disorders, lymphangioma

Lymphatic malformations (LMs) are rare vascular malformations both in humans and animals. However, in recent years, concern about these malformations in humans has increased.

When describing vascular anomalies the nomenclature is confusing. Some authors classified lymphangiomas as being: capillary (or simplex), cavernous, and cystic lymphangiomas (Yildirim et al. 2004; Wiegand et al. 2008). There are other classifications based on morphological aspects and they are divided into: macrocystic, microcystic and combined lymphagiomas (Wiegand et al. 2008). Currently, LMs are classified into two categories: "vascular tumours" and "vascular malformations" based upon clinical, histological and biochemical differences (Wiegand et al. 2008; Perkins et al. 2010). It has not been formally demonstrated that LMs are malignant disorders, and despite their unclear aetiology, some observations suggest a genetic aetiology because up to $40 \%$ of LMs detected during foetal development occur in children who present chromosomal anomalies (Perkins et al. 2010).

In cattle, LMs have been described and a hypothesis of a genetic disorder transmissible through an autossomal recessive gene was also established (Wanke et al. 1990). These authors described the clinical case of two calves showing cervical hygroma (macrocystic LMs) at birth and they concluded that analysis of the pedigrees indicates that the lymphocysts may represent a hereditary malformation.

As this is an unknown and rare case in ruminants, the aim of this work was to provide veterinary practitioners and researchers with some knowledge achieved throughout the study of this clinical case.

Address for correspondence:

Dr. Delia Lacasta

Animal Pathology Department

Veterinary Faculty, University of Zaragoza

Miguel Servet 177, 50013 Zaragoza, Spain 


\section{Clinical report}

A five-month-old Rasa Aragonesa male lamb with a history of cervical protuberances occurring from birth was received at the Ovine Clinical Service of the Veterinary Faculty of Zaragoza, Spain. The animal showed three round swollen lumps parallel to the trachea on the left side of the neck (Plate IV, Fig. 1). According to the farmer, the round nodules were increasing proportionally to the animal development since birth, but they did not interfere with the viability of the lamb, although poorer development was observed in comparison with other animals of the same age. Physical examination revealed normal findings. On palpation, non-painful lumps showed homogenous transilluminable liquid contained in separated structures ranging from 52 to $12 \mathrm{~cm}$ of diameter. Clinical examination also showed an enlargement of the left pre-scapular lymph node. Blood samples were taken for haematological analyses and no abnormalities were found. Ultrasound confirmed a multicystic lesion with internal separations and no blood flow was detected on the colour flow Doppler.

Samples of this fluid content were taken for analysis of physical and histological features. It was a clear, aqueous, proteinaceous fluid ( $>500 \mathrm{mg} / \mathrm{dl})$ of about $80 \mathrm{ml}, \mathrm{pH} 8.0$, density 1010 and nitrite and glucose traces. After 5 min at $1491 \times g$ centrifugation, a smear stained with haematoxilin-eosin showed no detection of lymphocytes and macrophages or infective organisms. Fluid was determined as lympha and protuberances were diagnosed as lymphatic malformations (LMs).

A surgery was accomplished in order to clarify the origin of the LMs. After conventional anaesthesia with xylazine $2 \%$ (Seton $2 \%$, Calier, Spain) at the dose of $1.5 \mathrm{ml} / 100 \mathrm{~kg}$ inj. i.v. and lidocaine $10 \mathrm{mg}$ (Anesvet, Ovejero, Spain) at the dose of $0.5 \mathrm{ml}$ inj. s.c., a circular incision was performed and all subcutaneous tissue over the external jugular vein was removed until its complete elimination. Histological evaluation of the tissue revealed dilated lymphatic vessels and channels in the dermis and hypodermis (Plate IV, Fig. 2). Some lymphatic vessels were filled with amorphous proteinaceous material and occasional lymphocytes and macrophages. They varied in size and were lined with thin endothelial cells. Several larger lymphatic channels presented alterations in their tunica media that consisted of abundant smooth muscular cells. A small amount of these cells located closer to the endothelium showed amphophilic coloration and necrosis. The stromal tissue exhibited disorganized bundles of collagenous and smooth muscle fibres together with few aggregates of lymphoid cells.

After a few days, humane sacrifice with sodium pentobarbital (Dolethal, Vetoquinol, Spain) at the dose of $10 \mathrm{ml}$ inj. i.v., was performed to carry out a deep anatomic and histopathological study. The tracheal ducts were dilated and the left pre-scapular lymph node was enlarged with a pale and wet cut surface. The protuberances were diagnosed as congenital lymphatic malformations.

\section{Discussion}

In ruminants, clinical cases of malformations in the lymphatic system have rarely been described. Some cases in bovine neonates (Wanke et al. 1990; Cruz et al. 1995) and a bilateral lymphangiomatous testicular lesion were described in a lamb (Brown et al. 2008). However, in the present clinical case, the location of the lesion was in the neck area. This is in concordance with lymphatic malformations in humans that occur most commonly in children, and two-thirds of all reported cases are found in the head and neck (Grasso et al. 2008; Wiegand et al. 2008; Colbert et al. 2013).

Lymphatic malformations are recognized as congenital malformations of the lymphatic system and are thought to originate from the sequestration of lymphatic tissue during the 
development of the lymphaticovenous sac (Colbert et al. 2013). Wanke et al. (1990) described two clinical cases in newborn calves with the same genetic origin. They established the hypothesis of the existence of a recessive autossomic gene transmitted by the bull. In our study, genetic origin could not be proved as it was the first case described on the farm and no other followed later.

Clinical and histopathological findings revealed very similar findings to those described in human LMs, except for metaplasic changes in the tunica media of the larger vessels, and the distention of the medullar sinus of the lymph node next to the lesion. These two findings could be considered as new clues to the study of the pathogenesis of these malformations. The present clinical case provides the first description of LMs in lambs in a case very similar to the human lymphatic malformations. This could be useful to improve the study of the pathogenesis of the disease.

\section{Acknowledgments}

The authors would like to thank Eleanor Harper for her assistance with English grammar review of the present paper.

\section{References}

Brown P, Smith K, Bazely K, Glover M, Barr F 2008: Bilateral lymphangiomatous testicular lesions in a lamb. Reprod Dom Anim 43: 246-248

Cruz AM, Riley CB, MacDonald DG, Ferguson JG 1995: Use of mesenteric lymphangiography in a calf with chylothorax and chyloperitoneum. J Am Vet Med Assoc. 206: 1567-1571

Colbert SD, Seager L, Haider F, Evans BT, Anand R Brennan PA 2013: Lymphatic malformations of the head and neck - current concepts in management. Br J Oral Maxillofac Surg 51: 98-102

Grasso DL, Pelizzo G, Zocconi E, Schleef J 2008: Lymphangiomas of the head and neck in children. Acta Otorhinolaryngol Ital 28: 17-20

Perkins JA, Manning SC, Tempero RM, Cunningham MJ, Edmonds JL Jr, Hoffer FA, Egbert MA 2010: Lymphatic malformations: current cellular and clinical investigations. Otolaryngol Head Neck Surg. 142: 789-794

Wanke VR, Distl O, Schmidt P, Hermanns W 1990: Cervical hygroma in the calf as an absolute cause of dystocia. An unusual malformation possibly of hereditary genesis in Braunvieh x Brown-Swiss breeds (in German). Berl Munch Tierarztl Wochenschr 103: 18-22

Wiegand S, Eivazi B, Barth PJ, von Rautenfeld DB, Folz BJ, Mandic R, Werner JA 2008: Pathogenesis of lymphangiomas. Virchows Arch 453: 1-8

Yildirim E, Dural K, Kaplan T, Sakinci U 2004: Cystic lymphangioma: report of two atypical cases. Interact Cardiovasc Thorac Surg 1: 63-65 
Plate IV

Santos N. et al.: Lamb congenital... pp. 239-241

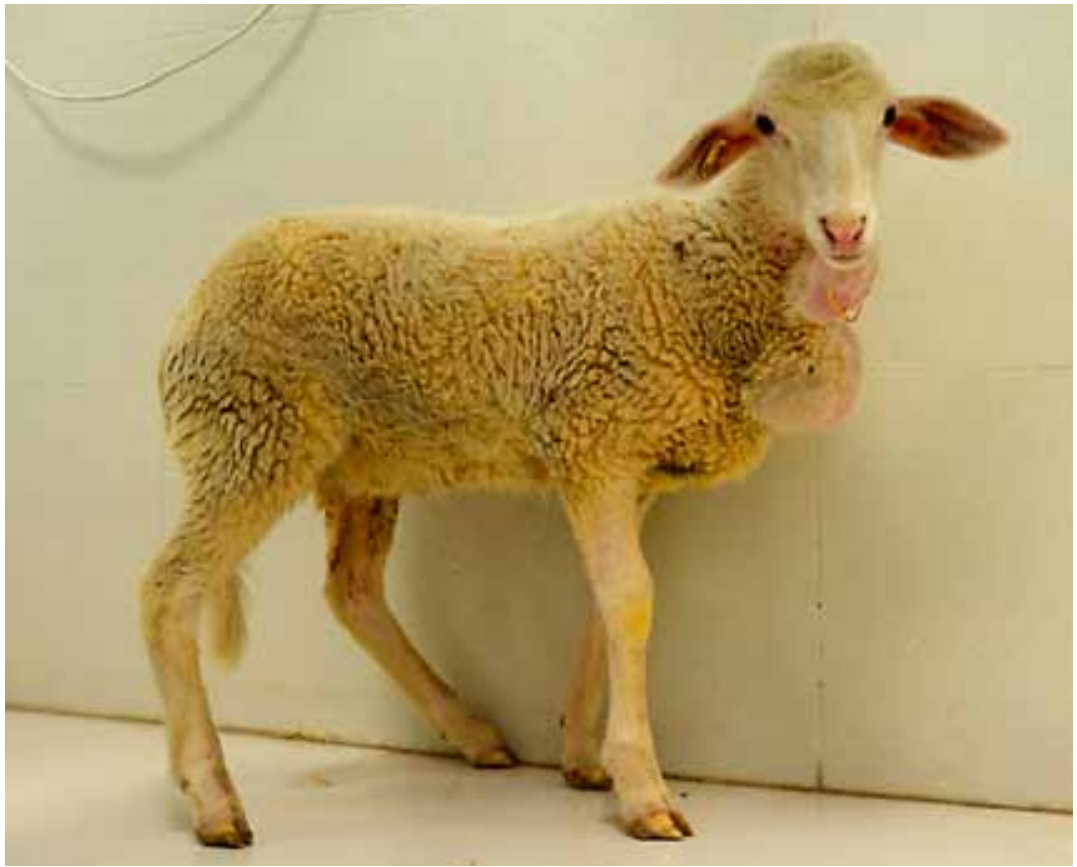

Fig. 1. Five-month-old lamb with cervical protuberances.

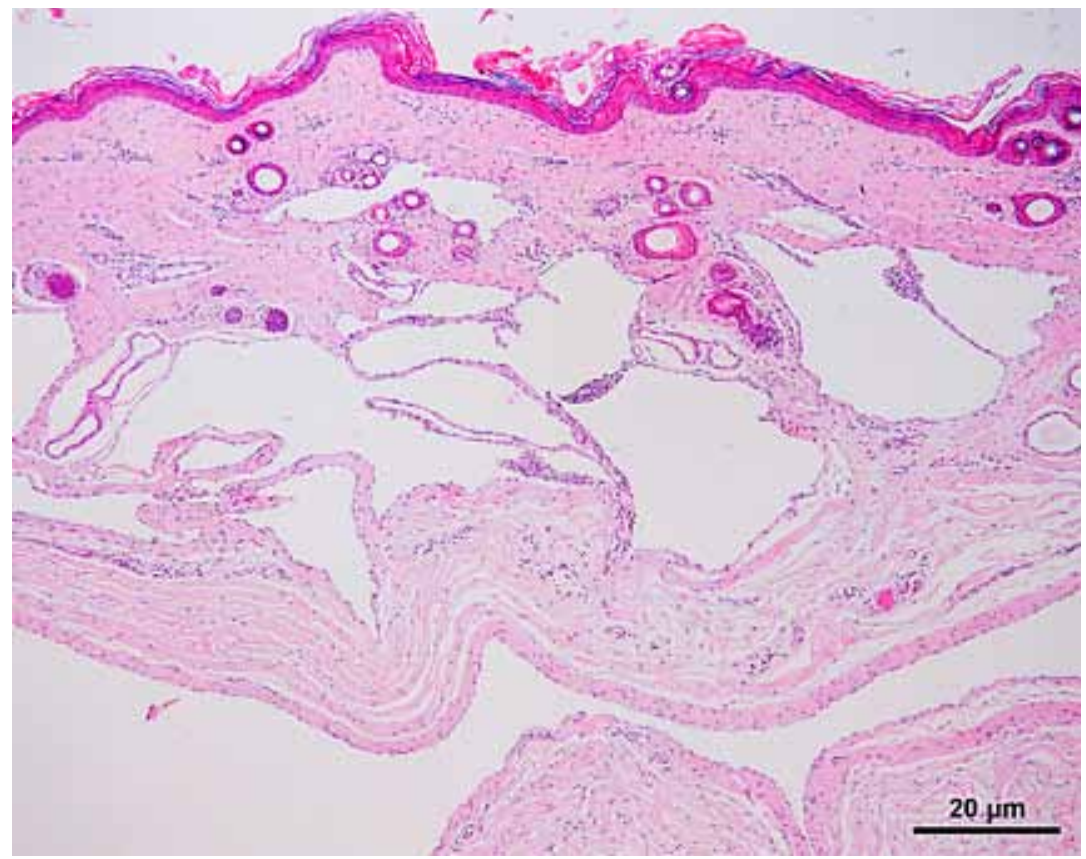

Fig. 2. Dilated lymphatic vessels and channels in a five-month-old lamb. Haematoxylin-eosin staining, $\times$ 40. Bar: $20 \mu \mathrm{m}$. 